\title{
La divulgación ambiental frente al cambio climático en la provincia de Camagüey, Cuba
}

\author{
MSc. Tel Pino SosA ${ }^{1}$ \\ Universidad de Camagüey, Cuba \\ Dra. C. Mayra Emeria Gonzalez Díaz ${ }^{2}$ \\ Centro de Investigaciones de Medio Ambiente de Camagüey. Cuba
}

Recibido: 21/08/2014

Aceptado: 25/01/2014

\begin{abstract}
Resumen
El cambio climático está considerado como uno de los principales desafíos a nivel global en los momentos actuales; lo que impone que se incrementen las medidas de adaptación y mitigación, en correspondencia con los peligros, el grado de vulnerabilidad y los riesgos a corto, mediano y largo plazos, dependiendo de las características de cada lugar; de ahí que en esta dirección la publicidad no escape a los cambios del entorno. Por el contrario, debe pensarse con mucha agudeza qué hacer. El presente trabajo aborda el papel que pueden desempeñar los medios de comunicación masiva en la divulgación de temas ambientales, profundiza en las mediaciones estructurales organizativas, cognitivas y los factores externos en el tratamiento de la información, con énfasis en el abordaje de los desastres naturales y los efectos del cambio climático, en su relación con los receptores o públicos hacia los cuales se dirigen los productos comunicativos y el contexto en el que se realizan estas acciones. Se sintetizan resultados de una investigación descriptiva orientada hacia la caracterización cualitativa del proceso de producción de los medios de difusión.

Palabras clave: mediaciones, productos comunicativos, receptores, contextos, cambio climático.
\end{abstract}

\section{Environmental dissemination to climate change in Camagüey province, Cuba}

\begin{abstract}
Climate change ranks as one of the major global challenges at the present time, which dictates the intensification of adaptation and mitigation measures, according the degree of vulnerability and risk in the short, medium and long periods, depending on the characteristics of each place; hence advertising in this direction not escape the changing environment, in contrast with much acuity should think what to do. This paper addresses the role of the media in the dissemination of environmental issues, explores organizational and structural mediations, the cognitives and the external factors in information processing with emphasis on addressing natural disasters and the effects of climate change in their relationship with the receiver or public to which the communication products and the context in which these actions are performed are headed. Results of a descriptive qualitative research-oriented to the characterization of the production process of the media are synthesized.

Keywords: mediations, communication products, receptors, contexts, climate change.

1 Tel Pino Sosa, Licenciado en Periodismo, Máster en Ciencias de la Comunicación, MSc. en Marketing y Gestión Empresarial. Decano de la Facultad de Comunicación y Lenguas de la Universidad de Camagüey. Cuba. E-mail: tel.pino@reduc.edu.cu

2 Mayra Emeria Gonzélez DíAz, Licenciada en Matemática, Dra. C. Agrícolas. Especialista del Centro de Investigaciones de Medio Ambiente de Camagüey. Cuba. E-mail: mgdcamaguey@ gmail.com
\end{abstract}




\section{Introducción}

El tiempo transcurrido desde la Conferencia de las Naciones Unidas sobre Medio Ambiente y Desarrollo en Río de Janeiro, 1992, se ha caracterizado por el continuo deterioro de la calidad ambiental mundial y la agudización de los principales problemas socioeconómicos internacionales.

Son cada vez más evidentes algunos de los problemas ambientales globales que más preocupan a la humanidad, entre ellos: la pérdida de la diversidad biológica; el deterioro de la capa de ozono; la contaminación urbana; el tráfico transfronterizo de desechos peligrosos; la contaminación de mares, océanos y zonas costeras; la degradación ambiental asociada a las condiciones de subdesarrollo y pobreza en que vive una parte significativa de la población mundial; así como los efectos ya visibles del cambio climático, derivados de inadecuados patrones de producción y consumo, el efecto de las guerras. Acciones, en su mayoría provocadas por el hombre, que agudizan la acumulación de gases de efecto invernadero en la atmósfera y contribuyen al deterioro del planeta.

Las sociedades humanas transforman y usan sus sistemas ambientales naturales con mayor o menor racionalidad, en dependencia de la organización social existente, del esquema de valores que predomine y de la normativa ambiental establecida. Además, las respuestas de las personas para satisfacer sus necesidades no serán las mismas en diferentes países, regiones, localidades y sectores; lo cual irremediablemente tendrá una implicación en el estado del medio ambiente.

Alcanzar la sostenibilidad ambiental constituye un reto para los países en desarrollo, lo que requerirá grandes transformaciones políticas, económicas y sociales, algunas de las cuales para Cuba son un hecho materializado y otras se encuentran en franco proceso de avance, teniendo como objetivo central la elevación de la calidad de vida del hombre, dado el carácter esencialmente humanista del proyecto social del país.

De acuerdo a las prioridades que establece la Estrategia de Ciencia e Innovación Cubana en el Área IV, entre los problemas cardinales del desarrollo sostenible del país y de la sociedad cubana -en los que la ciencia, la tecnología y la innovación desempeñan un papel significativo-, se destacan: ${ }^{3}$

- Problemas del desarrollo sostenible

- Adaptación al cambio climático

- Conservación y utilización racional de los recursos naturales del país, en particular, suelos, bosques, agua y biodiversidad.

- Vulnerabilidad y riesgos de desastres naturales y sanitarios

Por su parte la Estrategia Ambiental Nacional ${ }^{4}$ materializa en Cuba la Agenda 21 y representa una contribución importante al desarrollo sostenible, en particular por el

${ }^{3}$ http://www.atenas.inf.cu/?wpfb_dl=249. Estrategia Nacional de Ciencia, Tecnología e Innovación 2011 - 2015. Síntesis. CITMA. 2010.

${ }^{4}$ Estrategia Ambiental Nacional. CITMA. 2010.www.medioambiente.cu 
papel decisivo del Estado y las ventajas de una economía planificada, con capacidad para proyectar de forma armónica y a largo plazo, el uso de los recursos. En este empeño también la Estrategia Nacional de Educación Ambiental (ENEA) reconoce entre sus objetivos el de "Fortalecer los procesos de comunicación ambiental para el desarrollo de capacidades en los medios de comunicación, comunidades e instituciones que contribuyan a la sensibilización y toma de conciencia de la población cubana“. ${ }^{5}$

La problemática asociada a este tema se reconoce por la propia ENEA: “... los realizadores de los medios de comunicación masiva no cuentan con la preparación necesaria para abordar las relaciones ambientales y su problemática en el país. Por otro lado, es insuficiente tanto el ejercicio de la crítica en los medios de comunicación masiva ante la actuación irresponsable y las afectaciones al medio ambiente, así como la difusión de buenas prácticas ambientales para la promoción de la sensibilidad ciudadana e institucional. Las vías que se utilizan, son muy planas e indirectas, sin debate, con información que no siempre logra llegar a los niveles culturales de la base."

De ahí que se propusiese la realización de una investigación con el objetivo de analizar cómo condicionan las mediaciones, el tratamiento de la información medioambiental en los medios de difusión de la provincia de Camagüey, en particular en el Periódico Adelante y la emisora Radio Cadena Agramonte, con énfasis en los temas de desastres y enfrentamiento al cambio climático.

\section{Desarrollo}

\subsection{Referentes teórico - metodológicos que sustentan la investigación}

La investigación es de tipo Comunicológica, de corte teórico - aplicada. Está orientada hacia la caracterización cualitativa del proceso de producción de los medios de difusión de la provincia de Camagüey (prensa escrita y radio) respecto al tratamiento de los temas ambientales, con énfasis en el contexto del cambio climático. Es descriptiva y deviene en explicativa - interpretativa.

El trabajo se centró en las mediaciones estructurales organizativas (aquí también las tecnológicas), cognitivas y los factores externos en el tratamiento de la información medioambiental con énfasis en el tratamiento de los desastres naturales y los efectos del cambio climático.

\subsubsection{Acerca de las mediaciones y el proceso de recepción}

García, 2004 ${ }^{6}$ define las mediaciones como la actividad que impone límites a lo que puede ser dicho y a las maneras de decirlo, por medio de un sistema de orden.

${ }^{5}$ Estrategia Nacional de Educación Ambiental 2011 - 2015. CITMA. www.educambiente. co.cu

${ }^{6}$ García Luis, J. (2004): La regulación de la prensa en Cuba: Referentes morales y deontológicos. La Habana: Universidad de La Habana. Tesis para optar al título de Doctor en Ciencias de la Comunicación. 
Martín Serrano ${ }^{7}$ (2008) aportó los fundamentos teóricos para estudiar el papel de la comunicación institucionalizada y los sistemas de mediación, que a nivel cognitivo reducen la disonancia entre la innovación tecnológica, el cambio cultural y la organización social. Sánchez $\operatorname{Ruiz}^{8}$ (1991:40) explica que la mediación es un factor que en mayor o menor medida determina la acción de un actor o actores sociales en diferentes niveles y repercute en el desarrollo consciente, racional y creativo.

Gámez (2007:22) $)^{9}$ declara que el paradigma de la mediación es quizá el más pertinente para la comunicación pública, pues esta juega un papel fundamental en el control social, ya que en ella pueden distinguirse claramente las instituciones mediadoras (los medios), los objetos mediadores (productos comunicativos) y los modelos mediadores (las representaciones contenidas en esos productos)

La noción de mediación es concebida como «proceso estructurante que configura y reconfigura, tanto la interacción de los auditorios con los medios, como la creación por el auditorio del sentido de esa interacción» $[\text { Orozco, 1993: 61 }]^{10}$. El autor sugiere cuatro grupos de mediaciones fundamentales y considera que la cultura las impregna a todas. Estas mediaciones son la individual, la situacional, la institucional y la tecnológica.

- Individual. Son las que surgen del sujeto (mediación cognoscitiva y mediación de referencia).

- Situacional. Son los escenarios y situaciones concretas en que se entabla la relación entre el espectador y el medio de comunicación y que se expresa a través de todas las otras mediaciones.

- Institucional. Son las que desempeñan las diferentes instituciones sociales de pertenencia de los sujetos, que al aportar un conjunto de recursos materiales y de poder, reglas y significados compartidos, crean una subcultura propia a través de la cual se realiza la mediación.

- Tecnológica. Son las que se derivan del medio de comunicación en tanto institución social, dispositivo electrónico, especialmente de la especificidad del lenguaje del medio, su grado de verosimilitud y representacionalismo. Aquí se incluye el papel de los géneros periodísticos.

Al valorar la obra de Orozco se puede apreciar que su perspectiva de la mediación múltiple constituye una propuesta desarrollada de operacionalización de las teorías latinoamericanas de la recepción, al permitir enlazar las reflexiones más abstractas con

7 Martín Serrano, M. (2008): La mediación social. Madrid: Akal.

8 SÁnchez Ruiz, E. (1991): "Apuntes sobre una metodología histórico- estructural (con énfasis en el análisis de medios de difusión)”, Comunicación y Sociedad, n 10-11, pp. 11-49.

9 Gámez Torres, N. (2007): “El paradigma de la mediación: crítica y perspectivas",Mediaciones Sociales, $\mathrm{n}^{\circ}$ 1, pp. 195-213. Disponible en http://www.ucm.es/info/mediars/MediacioneS1/ Indice/GamezTorres/assets/195-213\%20Gamez\%20Torres.pdf. Consultado mayo 2014.

10 Orozco, G.: "Hacia una dialéctica de la recepción televisiva: la estructuración de estrategias por los televidentes", en Comunicaçao \& Politica na América Latina, CBELA, Año XII, nos. 22-25, 1993, pp. 57-73. 
los receptores reales a través de estudios empíricos, lo cual explica la gran cantidad de análisis que en el continente han inspirado este enfoque.

El principal aporte del modelo es la integración que permite entre los niveles teórico y empírico del conocimiento, lo que constituye uno de los mayores retos metodológicos de la investigación social. Entre sus méritos se cuentan la comprensión dialéctica y flexible del proceso de recepción y su énfasis en los determinantes socioculturales.

Las teorías y enfoques actuales de la recepción muestran una gran riqueza y diversidad, y aunque en sus rasgos principales exhiben ante todo coincidencias, presentan también algunas diferencias sustanciales, dadas esencialmente en:

1. La mayor o menor preminencia que en la estructuración de las audiencias otorgan a los factores macrosociales, económicos y a las clases sociales.

2. El nivel más o menos teórico o empírico en que se desarrollan.

3. Las fuentes teórico-metodológicas de que se nutren.

4. Las categorías empleadas para la integración de los procesos macro y microsociales

5. El modo de entender las mediaciones que en el proceso de recepción intervienen.

Según Alonso, M. opinión compartida por el autor .... los enfoques de Ang ${ }^{11}$, Morley ${ }^{12}$, Murdock ${ }^{13}$ y Lull ${ }^{14}$ ofrecen una perspectiva de análisis pertinente para el estudio de la recepción desde una postura crítica y compatible con la comprensión materialista dialéctica de los procesos de consumo cultural."15

La propia autora señala que “... de especial valor resulta la preminencia otorgada a los aspectos macrosociales y a la dimensión económica en la estructuración de las audiencias, la inserción del proceso de recepción dentro de prácticas socioculturales más amplias, la necesidad de no diluir la problemática del poder, la hegemonía y las clases sociales - aun cuando la recepción no puede entenderse como predeterminada por dichos factores - y la visión flexible e integradora de las instancias macro y microsociales.

${ }^{11}$ ANG, I.: «Buscado: audiencias. Sobre las políticas de los estudios empíricos de audiencia», en Cuadernos de Comunicación y Cultura, no. 24, Facultad de Ciencias Sociales, Universidad de Buenos Aires, 1993,

12 Morley, D.: «La "recepción" de los trabajos sobre la recepción. Retorno a El Público de Nationwide», en Dayan, Daniel (comp.). En busca del público. Recepción, televisión, medios, Ed. Gedisa, Barcelona, 1997, pp. 29-48.

${ }_{13}$ Murdock, G.: «La investigación crítica y las audiencias activas», en Estudios sobre las Culturas Contemporáneas, vol. IV, no. 10, Universidad de Colima, México, 1990,pp. 187-230.

14 Lull, J.: «La estructuración de las audiencias masivas», en Diálogos de la Comunicación. (Perú) (32): 50-57, 1992.

15 Alonso, Margarita. Principales paradigmas en el estudio del proceso de recepción en la teoría de la comunicación de masas. Año 2009. 


\subsubsection{La formación del personal de la prensa en los temas ambientales. El periodismo ambiental}

Para formar a un periodista competente se precisa de una acertada capacitación técnica y profesional que no surge de la espontaneidad, el periodista de hoy requiere de una sólida preparación cultural, de una cosmovisión holística del mundo en que vive, si pretende entenderlo y actuar decididamente en su mejoramiento.

La formación de los periodistas está determinada también por la existencia de recursos materiales que se disponen para la capacitación; la reposición gradual y sistemática de los profesionales, de manera que se cuente con ellos en diferentes estadíos de madurez; el acceso a personal docente de prestigio en el sector y a planes de estudio actualizados; el fomento de vías alternativas de superación de forma que, junto con la formación académica se exploten otros espacios que permitan el acercamiento a las teorías y tendencias más novedosas y a los productos comunicativos; de ahí la necesidad de concebir de forma estratégica el desarrollo de cursos, diplomados, maestrías y doctorados, entre otras formas de superación.

Un aspecto de vital importancia lo constituye la comprensión de una formación permanente por parte de los cuadros decisores, quienes en no pocas oportunidades se desentienden de su propia superación, negando así el carácter dinámico de la labor periodística.

Al referirse a la necesidad de que el periodismo ambiental se ubique en el centro de los problemas que aquejan al planeta hoy, María Novo -catedrática UNESCO de Educación Ambiental y Desarrollo Sostenible de la UNED- ${ }^{16}$, en el discurso de apertura de la Conferencia Inaugural del X Congreso Nacional de Periodismo Ambiental (Madrid, 20 y 21 de noviembre de 2013) expresó: “....empezamos a ver como normales situaciones que son verdaderamente desastrosas para la naturaleza y para el medioambiente. Así que necesitamos dar un salto, un salto mental, epistemológico... Yo diría que un salto antropológico, incluso, para comprender, develar y explicar las raíces de nuestra crisis y de la insostenibilidad de nuestras formas de vida. Tenemos que plantearnos una mirada nueva, con ojos nuevos; y esto tiene un nombre: un cambio de paradigma."

"Es importante que las nuevas agendas de contenidos respondan a un modelo educativo, en el cual emisores y receptores interactúen e intercambien roles en un diálogo constructivo, en las mayores condiciones posibles de igualdad", precisa Mario Esquivel (2008: 424-425) y abunda en que se trata en esencia de una verdadera revolución en el periodismo, basada en la educación recíproca y simultánea de los actores situados a ambos lados del proceso comunicativo.

${ }^{16}$ Novo, María: El periodismo ambiental en tiempos de crisis. 22 mayo, 2014 | Filed under: APIA 


\subsection{Antecedentes que soportan la divulgación ambiental para la reducción de desastres y el enfrentamiento al cambio climático en Camagüey}

Al reflexionar sobre el papel que pueden desempeñar los medios de difusión en la divulgación de temas ambientales, en particular los relacionados con la reducción de desastres y el enfrentamiento al cambio climático resulta interesante profundizar en los receptores o públicos hacia los cuales se dirigen los productos comunicativos y el contexto en el que se realizan estas acciones.

Un antecedente de gran valor lo constituyen los estudios de percepción de los peligros de desastres en la provincia de Camagüey, cuyos resultados, si son tenidos en cuenta por decisores y por los medios de difusión masiva, harían una contribución a la preparación de la población (públicos o destinatarios de los mensajes) en todas las etapas del ciclo de reducción de desastres (prevención, preparación, respuesta y recuperación), favoreciendo la minimización de los impactos ambientales que puedan provocar estos eventos, con la consiguiente disminución de las pérdidas de vidas humanas y recursos materiales.

El tema se ubica en el centro de las prioridades de la política ambiental cubana. Los "Lineamientos de la política económica y social del Partido y la Revolución" aprobados en abril de 2011 dotan de un nuevo impulso a la política ambiental en Cuba, así lo refleja la Estrategia Ambiental Nacional (EAN) para el período 2011 - 2015; que, al contextualizar las acciones que deben ser acometidas, refleja que el panorama económico nacional se encuentra fuertemente influido por las implicaciones para el país de la crisis global comenzada a finales del 2008, con una sensible disminución de los ingresos proyectados, los impactos del aumento del precio de los alimentos - $60 \%$ más altos que a principios de la década de 2000 -, aunado a la pérdida de las cosechas por los huracanes que azotaron en 2008 y las ocasionadas por las prolongadas e intensas sequías de los años 2003-2005 y 2010.

Por otra parte se avizora que "...el cambio climático constituye el principal desafío global, que impone incrementar la aplicación de medidas, a corto, mediano y largo plazo, de adaptación y mitigación, en correspondencia a los peligros, el grado de vulnerabilidad y los riesgos a que está sometido nuestro archipiélago." ${ }^{.17}$

Por la Directiva $N^{\circ} 1$ del 2010, del Presidente del Consejo de Defensa Nacional ${ }^{18}$, se responsabiliza al Ministerio de Ciencia, Tecnología y Medio Ambiente (CITMA) con el apoyo del potencial científico del país, a la realización de Estudios de Peligro, Vulnerabilidad y Riesgo de Desastres para identificar todas las zonas dentro del territorio nacional con posible afectación por peligros, con vistas a perfeccionar el proceso de

${ }^{17}$ Ministerio de Ciencia, Tecnología y Medio Ambiente. Estrategia Ambiental Nacional. $2011-2015$.

18 Directiva No. 1 del Vicepresidente del Consejo de Defensa Nacional para la planificación, organización y preparación del país para situaciones de desastre. La Habana. 2005 
reducción de desastres que dirige la Defensa Civil. Paralelamente, el Grupo Provincial creado a estos efectos, bajo la dirección de la Agencia de Medio Ambiente (AMA), realizó los estudios de percepción de los peligros hidrometeorológicos, con el objetivo de perfeccionar el enfrentamiento a inundaciones por intensas lluvias, penetraciones del mar y afectaciones por fuertes vientos.

Para la realización de dicho trabajo se realizó una revisión bibliográfica sobre los estudios que dan respuesta a la Directiva 1, las Estrategias Ambientales y Estudios de Percepción en el país y en la provincia, relacionados con el tema. Se tomó en consideración la Estrategia de Comunicación Social aprobada por el CITMA y los Lineamientos del Programa de Enfrentamiento al Cambio Climático, entre otros.

Se partió además de una caracterización de los medios de difusión masiva en la provincia, en cuanto al tratamiento de los temas ambientales, poniendo énfasis en los aspectos relacionados con la reducción de desastres, desde el análisis de las mediaciones que inciden y la perspectiva del estudio de los receptores, con una postura crítica de los procesos de consumo cultural.

Del universo de medios de difusión masiva con que cuenta la provincia se seleccionaron los provinciales: Periódico Adelante y Radio Cadena Agramonte. Se consideraron las potencialidades de la Universidad de Camagüey de insertarse como emisor de productos comunicativos a través del Periódico Digital “Avanzada”, el sistema de web administrado por el Centro de Gestión e Información de la Universidad, el papel de la radio base y la inserción de estudiantes de la carrera de Periodismo en los diferentes medios de difusión masiva en la provincia como parte del diseño curricular a través de prácticas laborales y mediante las colaboraciones de estudiantes y profesores a medios de prensa locales y nacionales.

\subsubsection{Principales resultados de los estudios de percepción de los Peligros hidrometeorológicos ${ }^{19}$}

El estudio se desarrolló en el año 2011. La muestra seleccionada alcanzó la cifra de 1.599 personas que representa el $0,20 \%$ de la población de la provincia. El 62,3\% de la muestra pertenece a los municipios de Camagüey, Florida, Nuevitas Vertientes y Santa Cruz del Sur. Esto indica el nivel de representatividad de cada uno de los municipios de acuerdo a su población y condiciones geográficas, económicas, sociales y ambientales. En la composición muestral a nivel de municipio se tuvieron en cuenta los mismos criterios que a nivel de provincia para cada uno de los Consejos Populares que lo integran.

En el proceso de entrevistas participaron los 13 especialistas municipales del Ministerio de Ciencia, Tecnología y Medio Ambiente, y 280 profesionales pertenecientes a las Sedes Universitarias Municipales del Ministerio de Educación Superior.

${ }_{19}$ Tomado del Informe "Estudio de percepción del peligro por intensas lluvias, inundaciones por penetraciones del mar y afectaciones por fuertes vientos en la provincia de Camagüey." Grupo Provincial de Riesgos. 2012. 
La valoración de las percepciones del peligro requiere una caracterización adecuada del contexto donde se desenvuelve la población. A continuación se resume la información imprescindible proveniente del estudio, con el objetivo de comparar la situación real de la provincia, los municipios y Consejos Populares con la percepción del peligro que tienen las personas:

- Los resultados del estudio de la percepción del peligro ante eventos hidrometeorológicos permite concluir que en la provincia, la percepción del peligro es predominantemente media en la mayoría de los encuestados (53\%) independientemente de las vulnerabilidades estructural, no estructural, funcional, económica, ecológica y social, existiendo comportamientos particulares a nivel de los Consejos Populares de cada municipio. Los municipios que presentaron más del $50 \%$ de encuestados en el grupo de percepción baja del peligro fueron Carlos Manuel de Céspedes y Sibanicú.

- La caracterización de la percepción del peligro por parte de la población incluye en el Grupo I, con una percepción alta del peligro, a las personas jóvenes, de nivel superior, trabajadores; campesinos o estudiantes, núcleos familiares de una o dos personas; pertenecientes al sector estatal y mixto, dirigentes y técnicos; en el Grupo II con una percepción media del peligro incluye a las personas del sexo masculino; adultos; sin escolaridad o de nivel medio y superior; trabajadores, jubilados y estudiantes; personas que residen en la comunidad desde menos de un año hasta tres años; núcleos familiares de personas solas hasta 4 personas que pueden no tener niños; ningún anciano hasta tres ancianos; pertenecientes al sector privado y mixto (77\%), dirigentes (61\%), trabajadores de servicios $(63 \%)$ y personas que residen en viviendas en condiciones buenas hasta regulares; y en el Grupo III, con una percepción baja del peligro, se encuentran las mujeres; adultos mayores; escolaridad de primaria a secundaria; amas de casa; jubilados; desocupados (53\%); personas con menos de un año de residencia; núcleos de más de cuatro personas; más de tres ancianos en el núcleo familiar (100\%); sector cooperativo; obreros, trabajadores administrativos y personas que residen en viviendas en malas condiciones.

Entre las principales recomendaciones que el estudio de percepción sugirió se encuentran:

- Socializar los resultados del estudio realizado a los decisores de acuerdo a su nivel de dirección.

- Introducir prácticas comunitarias participativas y focalizadas de gestión y educación ambiental con énfasis en los temas de peligro, vulnerabilidad y riesgos.

- Profundizar en el estudio de las creencias, actitudes, valores, conductas y comportamientos que favorecen la prevención y/o mitigación de los peligros hidrometeorológicos en comunidades seleccionadas por sus características.

- Potenciar el papel de los agentes socializadores escuela-familia-comunidad en el trabajo con los temas de peligro, vulnerabilidad y riesgos. 
- Capacitar a nivel local a los grupos meta identificados en este estudio utilizando las potencialidades tecnológicas de los Centros de Educación Superior que incluyan la integración de la capacitación presencial con la capacitación a distancia.

- Capacitar a los especialistas municipales relacionados con el tema para desarrollar acciones vinculadas al diagnóstico permanente de las percepciones del peligro como parte de la capacitación que desarrollan en sus territorios.

- Desarrollar talleres en las comunidades que presentan mayor vulnerabilidad social para informar y orientar acerca de los resultados del estudio que le competen.

Para el estudio de las acciones de divulgación ambiental frente al cambio climático se consideraron además las premisas siguientes:

- Poca calidad, diversidad y efectividad de los productos dedicados al medio ambiente en los medios de comunicación masiva y la preparación de los realizadores para este empeño, resultando una programación carente de atractivo y rigor, que no promueve la sensibilización ciudadana e institucional a través del ejercicio de la crítica ante las afectaciones al medio ambiente y la difusión de buenas prácticas.

- Diversas mediaciones de tipo estructural organizativo, cognitivas y externas pudieran obstaculizar la presencia y el tratamiento en profundidad de la información medioambiental, específicamente la relacionada con los desastres y los nuevos escenarios de cambio climático.

- Existen limitaciones en el empleo de recursos expresivos que permitan la elaboración de informaciones más amenas, ricas en matices que propicien una mayor eficacia del mensaje.

\subsubsection{Dimensiones del estudio sobre las mediaciones que intervienen en la efectividad} de la labor divulgativa en los medios para el enfrentamiento al cambio climático

Para el estudio de las mediaciones estructurales organizativas, la investigación se centró en el perfil editorial (ideología), la intencionalidad editorial, los libros de estilo, las rutinas productivas y las tecnologías del medio; en el caso de las mediaciones cognitivas se acentuó el análisis en la cultura e ideologías profesionales, el concepto de noticia, valores noticia y rutinas productivas de los actores; mientras que en la valoración de los factores externos el análisis estuvo enmarcado en las fuentes institucionales, las fuentes expertas, las fuentes populares y los funcionarios.

De gran valor para la investigación resultó el tratamiento que en los medios se brinda a la información medioambiental, para lo cual se tuvo en cuenta la prevalencia de estilos y géneros periodísticos, los recursos expresivos más utilizados, las estrategias de fuentes, los contextos que se utilizan y se profundizó en el análisis de los asuntos. 


\section{Consideraciones generales del estudio}

Se llegó a las siguientes consideraciones generales en los medios analizados:

- No cuentan con políticas comunicativas enfiladas a los temas medioambientales.

- Se evidencian enfoques inadecuados en el tratamiento de la información ambiental, con análisis muy someros de los temas, poco argumentados, poca profundidad de los mensajes, tendencia a enfatizar más en los efectos que en las causas, predominio de causas externas, bajo nivel de promoción de la participación y falta de sentido de responsabilidad de estos medios con la problemática.

- No existen diagnósticos de necesidades de capacitación del personal de los medios, en particular de los periodistas, referentes a la temática ambiental, a pesar del desconocimiento que muestran sobre aspectos claves de la materia.

- Se observan desajustes entre la información que se ofrece y la realidad contextualizada, siendo muy bajo el protagonismo de la población respecto a los sucesos noticiosos.

\section{Conclusiones}

1. Los resultados del trabajo investigativo posibilitaron conocer en profundidad las limitaciones y potencialidades que existen para realizar un periodismo más efectivo en el Periódico Adelante y en la emisora Radio Cadena Agramonte, respecto a la problemática asociada a los desastres y el enfrentamiento al cambio climático en Camagüey.

2. Se evidencia que los trabajos periodísticos asumen a los destinatarios como un público homogéneo, sin particularizar en las características socioculturales y el contexto en que se aborda la información.

3. En el tratamiento de la información generalmente es bajo el protagonismo de la población respecto a los sucesos noticiosos.

4. Se considera que existen debilidades en el manejo de la información, lo que contribuye a las bajas percepciones ambientales, en particular en lo que a desastres respecta, entre ellos: El hecho de que no existan políticas comunicativas en los medios de difusión estudiados respecto a los temas ambientales hace que el tratamiento de los mensajes esté marcado por la espontaneidad, a la vez que es débil la argumentación y el manejo de los géneros periodísticos.

\section{Recomendaciones}

1. Establecer políticas comunicativas dirigidas a los temas medioambientales en los órganos de prensa e implementar los resultados de los estudios de percepción con vistas a dosificar y dotar de intención los mensajes periodísticos, atendiendo a los determinantes socioculturales.

2. Capacitar a periodistas y profesionales que manejan la información en temas ambientales, en particular en lo referido a peligros de desastres y enfrentamiento al cambio climático, a partir de considerar los diagnósticos de necesidades de capacitación del personal.

Incorporar a los públicos receptores en el tratamiento de la información, dando mayor protagonismo a los diferentes estratos según las debilidades de las percepciones y el contexto provincial y local. 


\section{Bibliografía}

Alonso, Margarita. (2009): Principales paradigmas en el estudio del proceso de recepción en la teoría de la comunicación de masas. Facultad de Comunicación Universidad de La Habana.

ANG., I. (1993): «Buscado: audiencias. Sobre las políticas de los estudios empíricos de audiencia». Cuadernos de Comunicación y Cultura, no. 24, Facultad de Ciencias Sociales, Universidad de Buenos Aires. Argentina.

CITMA (2010a): «Estrategia Nacional de Ciencia, Tecnología e Innovación 2011 2015». Síntesis. http://www.atenas.inf.cu/?wpfb dl=249. Consultado 2 de marzo de 2012.

CITMA (2010b): «Estrategia Ambiental Nacional». 2011 -2015. La Habana. Cuba. www.medioambiente.cu. Consultado mayo 2014.

CITMA (2010c): «Estrategia Nacional de Educación Ambiental 2011 - 2015».www. educambiente.co.cu. Consultado 2 de marzo de 2012.

EMNDC. Estado Mayor Nacional de la Defensa Civil (2005): Directiva No. 1 del Vicepresidente del Consejo de Defensa Nacional para la planificación, organización y preparación del país para situaciones de desastre. La Habana. Cuba.

EMNDC. Estado Mayor Nacional de la Defensa Civil (2010): Directiva No. 1 del Presidente del Consejo de Defensa Nacional para la planificación, organización y preparación del país para situaciones de desastre. La Habana. Cuba.

GÁmeZ T., N. (2007): «El paradigma de la mediación: crítica y perspectivas», Mediaciones Sociales, $\mathrm{n}^{\circ}$ 1, pp. 195-213. Disponible en http://www.ucm.es/info/ mediars/MediacioneS1/Indice/GamezTorres/assets/195-213\%20Gamez\%20Torres. pdf. Consultado mayo 2014.

GARCía L., J. (2004): «La regulación de la prensa en Cuba: Referentes morales y deontológicos». Tesis para optar al título de Doctor en Ciencias de la Comunicación. Universidad de La Habana. Cuba.

Lull, J. (1992): La estructuración de las audiencias masivas. Diálogos de la Comunicación. Perú. (32): 50-57, 1992.

Martín S., M. (2008): La mediación social. Madrid: Akal.

Morley, D. (1997): «La "recepción" de los trabajos sobre la recepción. Retorno a El Público de Nationwide». En Dayan, Daniel (comp.). En busca del público. Recepción, televisión, medios, Ed. Gedisa, Barcelona, 1997, pp. 29-48.

MuRdock, G. (1990): «La investigación crítica y las audiencias activas». Estudios sobre las Culturas Contemporáneas, vol. IV, no. 10, Universidad de Colima, México, pp. 187-230.

Novo, María. (2014): El periodismo ambiental en tiempos de crisis. 22 mayo, 2014. Filedunder: APIA

Orozco, G. (1993): «Hacia una dialéctica de la recepción televisiva: la estructuración de estrategias por los televidentes», en Comunicaçao \& Politicana América Latina, CBELA, Año XII, nos. 22-25, 1993, pp. 57-73.

SÁNCHEZ, R. E. (1991): «Apuntes sobre una metodología histórico- estructural (con énfasis en el análisis de medios de difusión)», Comunicación y Sociedad, $\mathrm{n}^{\mathrm{0}}$ 10-11, pp. 11-49. 
Anexo 1

\section{Guía para la realización del diagnóstico en los medios de difusión masiva}

Periódico Adelante y Radio Cadena Agramonte Medio de Difusión:

\section{Objetivo del trabajo}

Conocer acerca del tratamiento que ofrece el órgano de prensa a la información medioambiental, con énfasis en el enfrentamiento a los desastres e impactos del cambio climático.

\section{Caracterización del medio de prensa desde el punto de vista estructural y organizativo}

a. Breve descripción de aspectos fundacionales (fecha de la primera edición) e históricos asociados al medio

b. Tecnologías (equipamiento digital y general, acceso a redes, etc.)

c. Libros de estilo (aspectos de interés según el objetivo del trabajo)

d. Rutinas productivas

e. Perfil editorial

f. Cantidad de periodistas

g. Periodistas especializados en medio ambiente:

Sí

No

II. Frecuencia mensual con la que se aborda el tema ambiental en el perí́dico por tipo de trabajo, recursos expresivos, fuentes y contextos

II.1. Cantidad de trabajos sobre medio ambiente publicados

II.2. Cantidad por tipo de géneros

II.3. Recursos expresivos más utilizados

II.4. Fuentes más frecuentes utilizadas: (\%) Institucionales, expertos, funcionarios y población.

II.5. Contextos más utilizados: Ecosistemas (zona costera, ciudad, áreas protegidas, cuencas hidrográficas, agroproductivos, etc.), empresas, fábricas, centros de servicios, sectores, otros).

III. Otros aspectos de interés según los objetivos del trabajo 